\section{Adaptation and counteradaptation to complex optical distortion*}

\author{
HELEN E. ROSS \\ University of Stirling, Stirling, Scotland \\ and \\ PETER LENNIE \\ Psychological Laboratory, Downing Street, Cambridge, England
}

The optical distortion caused by wearing a facemask in water magnifies the angular size of objects and reduces their optical distance. However, objects generally appear to be further than their optical distance, with the result that points in the left part of the visual field are apparently displaced to the left, and those on the right to the right. Experiments on hand-eye coordination under water showed that adaptation to one aspect of the distortion produced some counteradaptation to complementary aspects: adaptation to distance produced increased lateral distortion, and adaptation to one side of the lateral distortion produced increased distortion on the opposite side. Nevertheless, "trading" was incomplete, and some overall adaptation of the visual metric occurred.

When a diver wears a facemask in water, he suffers a complex optical distortion. Due to refraction of light at the air/glass/water interface, the image of an object under water is located at about three-quarters of its physical distance from the faceplate (Fig. 1). The distance distortion increases in the peripheral field of view, producing curvature distortion. The distortion also produces angular magnification, which ranges from about 1.33 for distant objects to 1.27 for close objects. (It is less for close objects, because the air space in the facemask is a significant proportion of the viewing distance.) The effect that the magnification has upon apparent size varies with the apparent distance-divers tend to locate objects further than the optical distance (Luria, Kinney, \& Weissman, 1967; Ross, 1967), and the overestimation of size increases systematically with the apparent distance (Ross, 1967). This would be expected from the size-distance invariance principle. At short distances in clear water, divers normally perceive objects as somewhere between the optical and physical distance, and as correspondingly enlarged. This was the situation in the experiments reported here. Figure 1 illustrates the discortion that occurs. The optical distance of all visual objects is reduced, and this reduction is greater the more peripheral the object: in Fig. $1, \mathrm{C}$ is

*This research was conducted at the U.S. Naval Submarine Medical Center, Groton, Connecticut, while the authors were supported financially by the American Institute of Biological Sciences. We should like to thank Dr. J. A. S. Kinney, Dr. S. Luria, Mrs. C. MacKay, Mr. F. Ward, and Mr. H. Katz for much practical help and useful discussion. We should also like to thank Mr. R. R. Macdonald for help with computing at Stirling University. reduced to $\mathbf{C}^{\prime}$, but $\mathbf{L}$ and $\mathbf{R}$ are reduced by a greater distance to $L^{\prime}$ and $\mathbf{R}^{\prime}$. If the apparent distance of objects is further than the optical distance, the apparent positions of $L^{\prime}, \mathbf{C}^{\prime}$, and $\mathbf{R}^{\prime}$ should lie along the line of sight to $L^{\prime}$, $\mathbf{C}^{\prime}$, and $\mathbf{R}^{\prime}$, but beyond them (e.g., at $\mathbf{L}^{\prime \prime}, \mathbf{C}^{\prime \prime}$, and $\left.\mathbf{R}^{\prime \prime}\right)$. An object at $\mathbf{L}$ under water will therefore appear to be nearer and further to the left than in air, and an object at $R$ nearer and further to the right. An object extending from $L$ to $R$ will obviously be magnified in angular size, and its apparent size will be enlarged (extending from $L^{\prime \prime}$ to $R^{\prime \prime}$ instead of $L$ to $R$ ). The lateral displacement of a point at $L^{\prime}$ or $R^{\prime}$ is thus equivalent to a size expansion, and it increases with the apparent distance. explanation.)
This distortion is complex, because some aspects of it are symmetrically opposite in the left and right halves of the visual field (and in the upper and lower halves). This is true of the angular distortion of the line of sight of peripheral objects: points on the left are displaced to the left, and points on the right to the right. The distance distortion is simpler, because it operates in the same direction over the whole of the visual field, even though it is more extreme in the periphery than in the center. The size distortion (and the lateral displacements which accompany it) is complicated in that it increases as the distance distortion is reduced, and vice versa. Some equivalent complications exist with prisms and other distorting systems, but they are rarely investigated. The most relevant studies are those of Kohler (1964), Pick and Hay (1966), and Pick et al (1969) on "gaze-contingent" distortion, which show that adaptation in two opposite directions is possible.

Previous underwater studies have shown that divers adapt visually to the distortions of curvature (Ross, 1970), size (Ross et al, 1970), and speed (Ross \& Rejman, 1972). Studies of hand-eye coordination under water have also shown adaptation to distance (Ono \& O'Reilly, 1971; Luria \& Kinney, 1970). Visual studies have shown a "trading" of size against distance adaptation (Franklin et al, 1970), those Ss who adapt to size tending to counteradapt to distance, and vice versa.

The present experiments were undertaken to investigate whether different training procedures

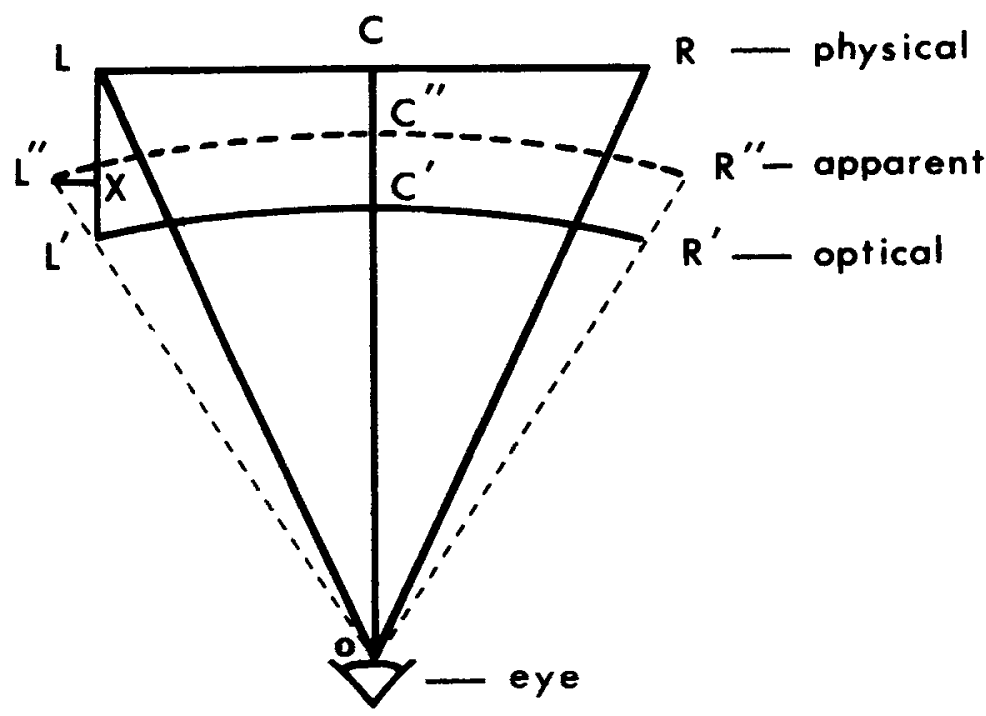

Fig. 1. Simplified diagram (not to scale) showing the effects of the optical distortion of the facemask on the apparent location of targets. (See text for 
differentially affected the amount of adaptation to different aspects of the distortion, as measured by a hand-eye coordination test. We were also interested to know whether, with appropriate training, lateral points would appear both further away and less displaced sideways (thus entailing a breakdown of size-distance invariance). The test involved a "ballistic movement" without visual guidance, a procedure recommended by Howard and Templeton (1966, Chap. 15) and Ono and O'Reilly (1971). The test was a development of the "placing" task used by Kinney et al (1970).

\section{METHOD}

Subjects

The Ss were 72 submarine trainees at the Naval Submarine Base, New London, Connecticut. Most Ss were in their early 20 s and had had little or no experience of scuba diving or snorkeling. Some were volunteers, and others were required to take part in the course of their naval duties.

\section{Apparatus and Procedure}

All testing took place on the grass beside an outdoor swimming pool, and in the pool $(20 \mathrm{ft}$ in diam and $4 \mathrm{ft}$ deep). The water temperature in the pool ranged from $70^{\circ}-90^{\circ} \mathrm{F}$. Ss wore a facemask for both the land and water tests and breathed through a snorkel for the water tests. Two identical tables, 24 in. square and 23 in. high, were used, one on the grass and one in the pool. A chinrest was clamped to the center of the near edge of the table, with the chinrest 32 in. above the ground. The $S$ knelt at the table with his chin on the chinrest, keeping his left hand at his side and his right hand on the table. He could not see his right hand when it was resting on the edge of the table, because the facemask restricted his field of view. A board ( $11 \times 22$ in.) was placed on the top of the table, the near end of the board being 8.75 in. from the near edge of the table. The board was checkered with black and white $1 / 2$-in. squares. Three black squares were marked with circles and were referred to as "left," "center," and "right." The central square was in the center of the board, 14.25 in. from the base of the chinrest. The other two squares were 4.5 in. to the left and right of the central square (measuring from center to center). When the $\mathrm{S}$ placed his chin on the chinrest and looked down at the central square, the distance between the center of the faceplate and the central square was about $13.5 \mathrm{in}$. The distance of the eye behind the faceplate was about 1.5 in. The average angle that the Ss' line of sight made with the surface of the board was $48 \mathrm{deg}$. Ss were asked always to keep their heads (and consequently faceplate) facing straight ahead at the central square and not to turn it to the left or right when looking at the other squares. This was because turning the mask would alter the optical effect considerably. For obvious safety reasons, it was not possible to clamp the S's head in the desired position. Most Ss succeeded in keeping their heads fairly still. However, some head movements did occur, and these were probably responsible for the slight deviations from the optical values which were found in the data.

S's task was to place a marker (a small metal stud, 1.13 in. high and $0.5 \mathrm{in}$. in diam) on one of the three target squares, without watching his hand. He glanced at the target square and then $E$ held a screen in front of his mask while he attempted to place the marker on the square from memory. He then withdrew his hand to the edge of the table, leaving the marker behind. On some trials ("feedback" trials), $E$ lifted the screen and allowed him to see where he had placed the marker. On other trials ("blind" trials), E returned the marker to S's hand before lifting the screen.

Each $S$ was tested first in air, and was given four trials at each of the three targets. The first two trials at each target were followed by feedback, while the second two were blind. (The purpose of giving feedback was to remove any constant errors before taking baseline measures. If this were not done, any later reduction in constant error would be confounded with changes due to perceptual adaptation.) The blind trials are referred to as the "Air 1" test. $S$ then entered the pool, wearing a facemask, snorkel, and weight belt. He knelt at the table with his chin in the chinrest and his face submerged, while breathing through the snorkel. He was tested as soon as possible after entering the water, with two blind trials on each of the targets, the targets being taken in the same order as in air. (This is referred to as the "Water 1" test). The six possible orders were used twice for each group of 12 Ss. Six groups of Ss were used, each being given a different training procedure. The first three groups were given 20 trials with feedback on the left, center, or right square only. The fourth group was given 20 blind trials on the center square. The fifth group was given 18 trials with feedback, using all three squares, in the sequence of two left, two center, two right (the pairs being in the same order as in the initial testing) repeated three times. The sixth group was given no specific training, but swam around the pool inspecting the bottom for the same length of time as the other training procedures ( $3 \mathrm{~min} 20 \mathrm{sec}$ ). Following the training procedure, $S$ was tested again without feedback for two trials on each square, in the same sequence as before ("Water 2" test). He was then led blindfold out of the pool, and removed his snorkel and weight belt. He knelt at the air table, opened his eyes, and was immediately retested with two blind trials on each square, in the same sequence " Air 2" test). (The purpose of keeping $S$ 's eyes closed in the air before testing was to minimize any fading of the aftereffect while he left the pool. For similar reasons, any Ss who were forced to surface in the middle of the test to clear their facemasks or snorkels were asked to keep their eyes shut above water.)

All trials were made at $10-\mathrm{sec}$ intervals, a second $E$ keeping time with a stopwatch, and recording the position of the marker for each trial (to the nearest $1 / 4$ in.) on a scaled chart.

The difference between the Air 1 and Water 1 tests gives a measure of the initial distortion experienced in water. The Water 1-Water 2 difference gives a measure of adaptation in water, and the Air 1-Air 2 difference gives a measure of the aftereffect in air. Most authors use the aftereffect as a measure of adaptation for practical and theoretical reasons (Rock, 1966). However, the adaptive shift in water is of more practical concern to divers, and may not necessarily equal the aftereffect (e.g., Kohler, 1964, p. 37; Ross et al, 1970; Welch, 1971).

\section{RESULTS AND DISCUSSION}

The mean positions in which the marker was placed in the different conditions are shown in Fig. 2. The diagrams also show the true (physical) position of the three targets, and the optical lcoation of the centers of the targets on the plane of the table (taking into account the $S$ 's angle of regard-see section on angular errors). The location of the marker can be considered in two different (but not independent) ways. In the first of these, the error can be expressed as displacements from the target square in the vertical (in depth) and lateral (horizontal) directions. These are the distances $L X$ and $L^{\prime \prime} X$ in Fig. 1 . Secondly, the position of the marker can be considered as an angular deviation from a line joining the S's eye to the central target (the angle $L^{\prime \prime} O C$ in Fig. 1). This measure is specified by the vertical and lateral displacements, but does not specify them. There are many combinations of vertical and lateral displacements which give the same angle. (Any point along the line OL" will specify the angle L"'OC. However, the 
experimental situation is more complex than this, because $S$ 's line of sight does not lie along the surface of the table but at an angle of $48 \mathrm{deg}$. The necessary calculations are described in the section on angular errors.)

Only two measures are necessary to specify the location of the marker. The vertical and lateral locations specify the angle, and the angle plus one of the other measures specifies the remaining measure. We use all the measures because they give rather different pictures of the adaptation process. The vertical and lateral measures specify the errors that occurred in relation to the target, while the angular measures enable us to say whether any adaptation occurred apart from the "trading" between the former measures.

Vertical Distance Errors

The position of the marker during the first water test deviated considerably from the optically predicted position. Ss placed the marker about midway between the optical and physical positions, showing approximately $50 \%$ compensation during the first water test. There was perhaps some slight adaptation over the six trials, but even the first trial was considerably less than the optical shift. The mean position on the first trial was 1.23 in. too near, while the mean optically expected position (over the three targets) was 2.25 in. Hay and Pick (1966) and Rock et al (1966) also found that Ss did not show the full optical displacement introduced by a prism.

It can be seen from Fig. 2 that all groups showed distance adaptation in the aftereffects (Air 2 further than Air 1), and all except the right group showed it in the water (Water 2 further than Water 1). The mean adaptive shift in water was 0.472 in. ( $t$ $=4.36, \mathrm{df}=71, \mathrm{p}<.0005$, one-tailed), which is equivalent to $39 \%$ adaptation from the mean initial error in water (1.20 in.). The mean aftereffect in air was 0.996 in. $(t=12.13$, df $=71$, $p<.0005$, one-tailed), which is $83 \%$ of the initial error. The aftereffects were about twice as large as the water shifts, and the difference was significant on an analysis of variance $(F=32.23, \mathrm{df}=1,420, \mathrm{p}<.001)$.

The analysis also showed significant differences between groups $(F=2.28$, $\mathrm{df}=5,420, \mathrm{p}<.05$ ), but no subcomparisons were significant using Tukey's method.

\section{Lateral Errors}

The mean lateral errors in the Water 1 test went in the expected directions (Fig. 1), but were asymmetrical in size. There was a small

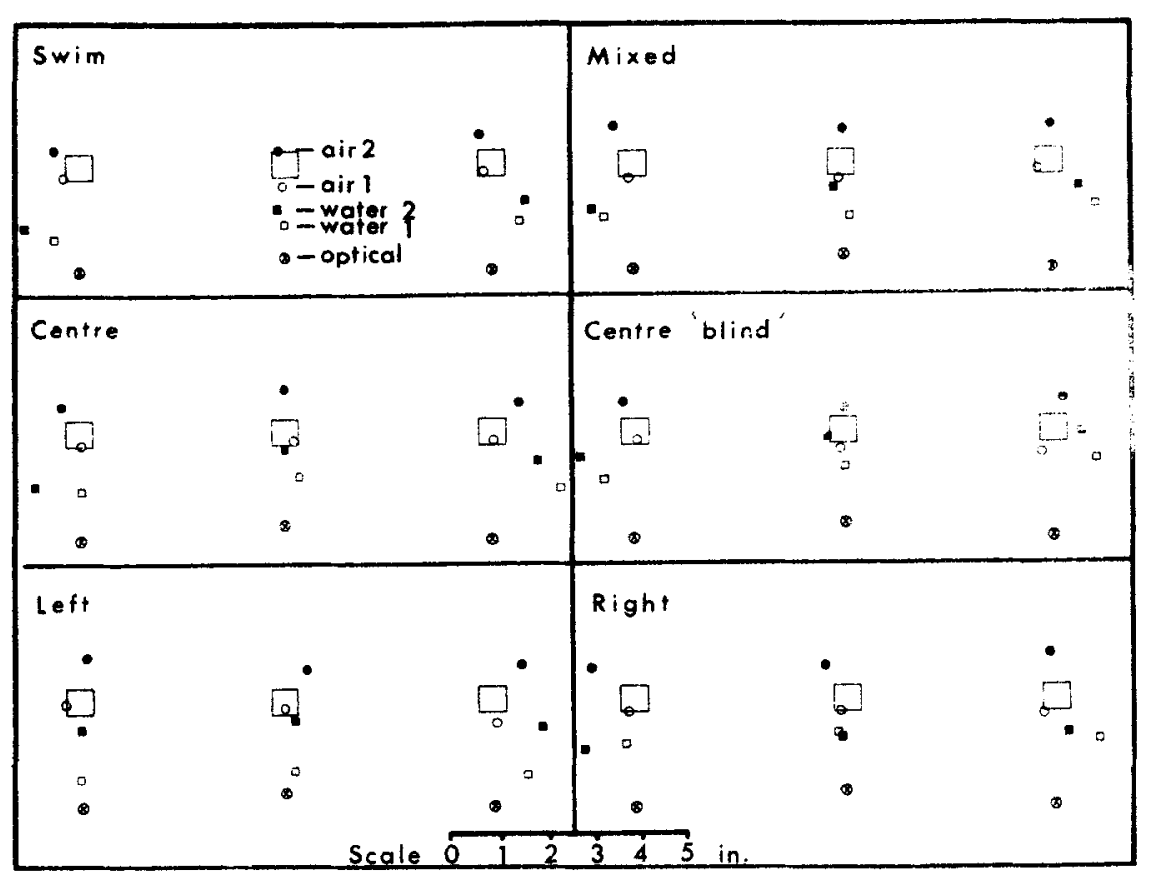

Fig. 2. Mean location of the marker in relation to the three targets for the different groups.

left error on the left target $(0.348 \mathrm{in}$.) but a large right error on the right target $(0.932 \mathrm{in}$.$) . There was also a$ slight right error on the central target (0.125 in.). Combining all three targets, there was a mean rightward bias of $0.236 \mathrm{in}$. ( $t=4.11$, df $=71$, $p<.001$, two-tailed). The rightward bias may perhaps be a consequence of the body and head turning to the left when the right hand is used; this would have the effect of increasing the optical distortion to the right and reducing it to the left.

The mean shifts in water (Water 2-Water 1 ) showed a leftward trend for all target positions (0.557 in. left; 0.244 in. center; 0.252 in. right). Combining all three targets, the mean leftward shift was 0.351 in. $(t=4.32$, $\mathrm{df}=71, \mathrm{p}<.001$, two-tailed). This shift can perhaps be explained as an adaptive shift from the right bias evident in the Water 1 test. Whatever its cause, it is not relevant to the main question of whether there is any adaptation to the lateral distortions caused by angular magnification. Adaptation would be indicated by a right shift on the left target and a left shift on the right target, and vice versa for counteradaptation. If the scores for the left and right targets are combined (counting all adaptive shifts as positive and all counteradaptive shifts as negative), there is a mean counteradaptive shift of 0.153 in. $(t=$ $1.68, \mathrm{df}=71, \mathrm{p}<.10$, two-tailed).

The mean aftereffects (Air 2-Air 1) showed no significant left or right bias, but clear counteradaptive shifts on the lateral targets. There was a left shift on the left target $(0.254$ in.), a right shift on the right target $(0.299$ in.), and a slight right shift on the central target (0.015 in.). Combining the left and right targets, there was a mean counteradaptive shift of 0.277 in. $(t=$ 5.85, df $=71, \mathrm{p}<.001$, two-tailed). An analysis of variance showed no significant difference between the size of the counteradaptive shifts in air and water $(F=3.10, \mathrm{df}=1,264)$. If the two are combined, there is a mean counteradaptive shift of 0.215 in. $(t=$ $4.34, \mathrm{df}=71, \mathrm{p}<.001$, two-tailed).

The different groups did not differ significantly from each other in the total amount of lateral counteradaptation. However, if the shifts are scored as left/right rather than as adaptive/counteradaptive, there is a clear difference between the direction of the shifts of the left and right groups $(t=2.76, \mathrm{df}=22$, $\mathrm{p}<.01$, one-tailed). As might be expected, these groups adapted on the trained side and counteradapted on the opposite side. Combining water shifts and aftereffects, the left group showed right shifts of 0.258 in. (left target), 0.242 in. (center target), and 0.373 in. (right target), while the right group showed left shifts of 0.841 in. (left target), 0.339 in. (center target), and 0.261 in. (right target). The mean right shift for the left group was 0.294 in. $(t=1.98, \mathrm{df}=11, \mathrm{p}<.05$, one-tailed), and the mean left shift for the right group was 0.480 in. $(t=1.94$, 
Table 1

Mean Angles Subtended at the Eye (Degrees)

\begin{tabular}{|c|c|c|c|c|c|c|c|c|}
\hline \multirow[b]{2}{*}{ Group } & \multicolumn{2}{|c|}{ Air 1} & \multicolumn{2}{|c|}{ Water 1} & \multicolumn{2}{|c|}{ Water 2} & \multicolumn{2}{|c|}{ Air 2} \\
\hline & Left & Right & Left & Right & Left & Right & Left & Right \\
\hline $\begin{array}{l}\text { Left } \\
\text { Right } \\
\text { Center }\end{array}$ & $\begin{array}{l}18.07 \\
17.72 \\
17.03\end{array}$ & $\begin{array}{l}18.28 \\
16.34 \\
17.07\end{array}$ & $\begin{array}{l}21.86 \\
19.70 \\
19.59\end{array}$ & $\begin{array}{l}23.85 \\
22.11 \\
25.32\end{array}$ & $\begin{array}{l}18.07 \\
23.44 \\
23.26\end{array}$ & $\begin{array}{l}21.59 \\
19.33 \\
21.47\end{array}$ & $\begin{array}{l}15.24 \\
18.81 \\
17.36\end{array}$ & $\begin{array}{l}17.67 \\
15.16 \\
17.75\end{array}$ \\
\hline $\begin{array}{l}\text { Center } \\
\text { (Blind) }\end{array}$ & 16.76 & 16.68 & 21.58 & 21.37 & 22.09 & 18.87 & 16.75 & 16.65 \\
\hline $\begin{array}{l}\text { Mixed } \\
\text { Swim }\end{array}$ & $\begin{array}{l}17.69 \\
18.39\end{array}$ & $\begin{array}{l}15.90 \\
16.42\end{array}$ & $\begin{array}{l}21.63 \\
23.66\end{array}$ & $\begin{array}{l}22.62 \\
21.98\end{array}$ & $\begin{array}{l}22.34 \\
24.46\end{array}$ & $\begin{array}{l}20.07 \\
20.82\end{array}$ & $\begin{array}{l}17.07 \\
18.21\end{array}$ & $\begin{array}{l}15.68 \\
15.07\end{array}$ \\
\hline Mean & 17.60 & 16.77 & 21.28 & 22.82 & 22.23 & 20.32 & 17.21 & 16.32 \\
\hline
\end{tabular}

df $=11, \mathrm{p}<.05$, one-tailed).

It seems clear that lateral adaptation can be achieved, given one-sided training, but only at the expense of the opposite side. Ss seem unable to adapt to two different directions of distortion, even when trained on both sides. Instead, there is a general tendency to counteradapt. This is predictable, if Ss adapt mainly to distance and the size-distance relationship remains unchanged. However, counteradaptation is compatible with a small change in the latter relationship. If there is a change in the right direction, some genuine adaptation has occurred; if not, Ss have merely traded one type of distortion for another. This problem is best investigated by converting the combined vertical and lateral shifts into angular measures, taking into account S's tilted viewing position.

\section{Angular Errors}

The vertical and lateral errors were combined to give the angular deviation of the marker from the central target, from the S's viewing point, according to the formula: $\tan \phi=$ $\sqrt{x^{2}+y^{2} \tan ^{2} 48 \operatorname{deg}} /(15+y$ sec $48 \mathrm{deg}$ ), where $x=$ lateral distance from central target, and $y=$ algebraic vertical distance from central target.

This formula takes into account the fact that S's line of sight made an angle of $48 \mathrm{deg}$ with the surface of the table, and that his eye was at a distance of 15 in. All angles are calculated as for viewing in air, the effect of refraction in water being considered later.

Table 1 shows the angles subtended by the left and right target settings for the different groups. The central setting is ignored, as deviations are small and uninformative (except in the case of the left and right groups, which show the shifts expected from the lateral measures). Table 2 shows the left and right angles combined, thus giving a measure of the total angular spread uncontaminated by lateral bias. It also shows the corresponding initial water effects, water shifts, and aftereffects. The initial angular increase in water varies a little from group to group, but the mean increase of $9.63 \mathrm{deg}$ is very close to the optical calculations. That is to say, the mean total angle for the Water 1 lateral targets (44.10 deg) is very close to the optically expected angle (43.65 deg). The latter angle is obtained by multiplying the Air 1 angle (34.37 deg) by 1.27 (the appropriate factor for very short viewing distances). The correspondence between the optical and obtained angles in water is probably as good as can be expected, taking into account the inevitable variation between $S s$ in the precise distance and angle of the faceplate. There is, thus, no evidence for any immediate adaptation on entering the water, though this had been implied by the vertical distance errors when considered in isolation from the lateral errors.

There was a mean adaptive shift in water of $1.55 \mathrm{deg}(t=4.04, \mathrm{df}=71$, $\mathrm{p}<.0005$, one-tailed) and an 71 , p <.05, one-tailed). These two measures were significantly different on an analysis of variance $(F=6.30, \mathrm{df}$ $=1,132, p<.025$ ). In this case, the aftereffect was smaller than the water shift, not larger as with the vertical and lateral measures. This shows the importance of considering the combined effect of the latter measures before drawing conclusions. If the vertical and lateral errors were considered without angular calculations, one might be tempted to conclude that no genuine adaptation had occurred: Ss simply "trade" one aspect of the visual field against another, such as left against right, or vertical distance against lateral errors. aftereffect of $0.84 \mathrm{deg}(\mathrm{t}=1.84$, df $=$
However, the mean angular measures show that there was some overall adaptation, as the angles were reduced with time. That is to say, even though "trading" undoubtedly occurs, it is not strictly proportional, and there is some overall gain in modifying the total visual field. Complete adaptation would entail an angular reduction of about $9 \mathrm{deg}$, so adaptation of $10 \%-17 \%$ was obtained in this experiment (the amount depending on whether the water shift or aftereffect is used as a measure).

An analysis of variance showed significant differences between groups $(F=3.32, \mathrm{df}=5,132, \mathrm{p}<.01)$ on the total angular adaptation; however, subcomparisons between groups using Tukey's method showed that only the left group differed significantly from the others. This group showed the most angular adaptation, but it is hard to find a reason for its superiority. The groups varied in the size of their initial error, and it is quite likely that this has some effect on subsequent adaptation. On the present evidence, it would not seem reasonable to conclude firmly that any training method is superior at producing overall adaptation. However, it is perhaps interesting that the center trained groups show on average very little adaptation; this is not surprising, since they had little opportunity to learn about the lateral errors. It is perhaps advisable to include some lateral training (or free swimming) to induce overall adaptation.

\section{GENERAL DISCUSSION}

Most studies of the effects of optical distortion report only adaptive changes. We want to point out that counteradaptive changes can also occur. The clearest example is perhaps the "trading" of one part of the visual field against another (e.g., left against right), or of distance against size (e.g., vertical against lateral errors). However, we do not wish to imply that "trading" is complete: if it were, there would be no "genuine" adaptation or effective restructuring of the whole visual metric. What we have in fact demonstrated is that both some trading and some overall adaptation occur.

Table 2

Total Angles (Degrees) Subtended at the Eye by the Left and Right Positions Combined

\begin{tabular}{lrrrrrrr}
\hline \multicolumn{1}{c}{ Group } & A1 & W1 & W2 & A 2 & W1-A1 & W1-W 2 & A1-A2 \\
\hline Left & $\mathbf{3 6 . 3 5}$ & 45.71 & $\mathbf{3 9 . 6 6}$ & $\mathbf{3 2 . 9 1}$ & $\mathbf{9 . 3 6}$ & $\mathbf{6 . 0 5}$ & $\mathbf{3 . 4 4}$ \\
Right & $\mathbf{3 4 . 0 6}$ & $\mathbf{4 1 . 8 1}$ & $\mathbf{4 2 . 7 7}$ & $\mathbf{3 3 . 9 7}$ & $\mathbf{7 . 7 5}$ & $-\mathbf{0 . 9 6}$ & $\mathbf{0 . 0 9}$ \\
Center & $\mathbf{3 4 . 1 0}$ & $\mathbf{4 4 . 9 1}$ & $\mathbf{4 4 . 7 3}$ & $\mathbf{3 5 . 1 1}$ & $\mathbf{1 0 . 8 1}$ & $\mathbf{0 . 1 8}$ & -1.01 \\
Center (Blind) & $\mathbf{3 3 . 4 4}$ & $\mathbf{4 2 . 9 5}$ & $\mathbf{4 0 . 9 6}$ & $\mathbf{3 3 . 4 0}$ & $\mathbf{9 . 5 1}$ & $\mathbf{1 . 9 9}$ & $\mathbf{0 . 0 4}$ \\
Mixed & $\mathbf{3 3 . 5 9}$ & $\mathbf{4 4 . 2 5}$ & $\mathbf{4 2 . 4 1}$ & $\mathbf{3 2 . 7 5}$ & $\mathbf{1 0 . 6 6}$ & $\mathbf{1 . 8 4}$ & $\mathbf{0 . 8 5}$ \\
Swim & $\mathbf{3 4 . 8 1}$ & $\mathbf{4 5 . 6 4}$ & $\mathbf{4 5 . 2 8}$ & $\mathbf{3 3 . 2 8}$ & $\mathbf{1 0 . 8 3}$ & $\mathbf{0 . 3 6}$ & $\mathbf{1 . 5 3}$ \\
Mean & $\mathbf{3 4 . 3 7}$ & $\mathbf{4 4 . 1 0}$ & $\mathbf{4 2 . 5 5}$ & $\mathbf{3 3 . 5 3}$ & $\mathbf{9 . 6 3}$ & $\mathbf{1 . 5 5}$ & $\mathbf{0 . 8 4}$ \\
\hline
\end{tabular}


Our results say nothing about the locus of adaptation. However, they do imply that Ss learn something more complex than an intellectual rule for correction (such as "Reach 1 in. further, and $1 / 2$ in. to the left or right"). The majority of Ss were unable to describe the nature of the distortion they had experienced in water, and some denied noticing any distortion. It is also unlikely that much proprioceptive adaptation occurred, since Ss could not see their own hands (except for the swimming group). It is more likely that our results reflect visual adaptation, Ss reaching where objects appear to be. It is not clear what type of information produced adaptation. Artificial feedback seemed to be no more effective than the same movements without feedback. Welch and Rhoades (1969) showed that knowledge of target-pointing error can be effective, but in their experiment $S$ s were allowed to glimpse the position of their fingers, while our Ss saw only the position of the misplaced marker. This difference may be critical. It is probable that our Ss made use of a less conscious type of feedback-the "rubbery" transformation of the visual field caused by small head movements. Lateral adaptation could be caused by paying more attention to one side of the field than to the other. It may also have been aided by Gibsonian adaptation, due to staring at a distorted checkerboard. The swimming group had less opportunity for Gibsonian adaptation, but more opportunity for movement and the sight of their own limbs.

On the practical question of the best method of training divers to adapt to the distortion, it seems clear that concentration on one aspect will tend to cause counteradaptation to some other aspect. It would seem wisest, then, either to give deliberate training to all aspects of the distortion or to encourage the diver to swim around and manipulate objects freely.

\section{REFERENCES}

FRANKLIN, S. S., ROSS, H. E., \& WELTMAN, G. Size-distance invariance in perceptual adaptation. Psychonomic Science, 1970, 21, 229.231.

HAY, J. C \& PICK, H. L. JR. Visual and proprioceptive adaptation to optical displacement of the visual stimulus. Journal of Experimental Psychology, $1966,71,150-158$

HOWARD, I. P., \& TEMPLETON, W. B. Human spatial orientation. New York: Wiley, 1966.

KINNEY, J. A. S., MCKAY, C. L., LURIA, S. M., \& GRATTO, C. L. The improvement of divers' compensation for underwater distortion. SMRL report, U.S Naval Submarine Base, Groton, Conn. 1970.

KOHLER, I. The formation and transformation of the perceptual world. Psychological Issues, 1964, 3, No. 4 (Monograph No. 12)

LURIA, S. M., \& KINNEY, J. A. S Underwater vision. Science, 1970, 167. 1454-1461.

LURIA, S. M., KINNEY, J. A. S., \& WEISSMAN, s. Estimates of size and distance underwater. American Journal of Psychology, 1967, 80, 282-286.

ONO, H, \& O'REILLY J, P. Adaptation to underwater distance distortion as a function of different sensory-motor tasks. Human Factors, 1971, 13, 133-140.

PICK, H. L., JR., \& HAY, J. C. Gaze-contingent adaptation to prismatic spectacles. American Journal of Psychology, 1966, 79, 443-450.

PICK, H. L., JR., HAY, J. C., \& MARTIN R. Adaptation to split-field wedge-prism s pectacles. Journal of Experimental Psychology, 1969, 80, 125-132.

ROCK, I. The nature of perceptual adaptation. New York: Basic Books, 1966.

ROCK, I., GOLDBERG, J., \& MACK, A. Immediate correction and adaptation based on viewing a prismatically displaced scene. Perception \& Psychophysics, 1966 , $1,351-354$.

ROSS, H. E. Water, fog and the size-distance invariance hypothesis. British Journal of Psychology, 1967, 58, 301-313.

ROSS, H. E. Adaptation of divers to curvature distortion under water. Ergonomics, 1970,13,489-499.

ROSS, H. E., FRANKLIN, S. S. WELTMAN, G., \& LENNIE, P. Adaptation of divers to size-distortion under water. British Joumal of Psychology, 1970, 61, 365-373.

ROSS, H. E. \& REJMAN, M. H. Adaptation to speed distortions under water. British Journal of Psychology, 1972, 63 (in press).

WELCH, R, B, Discriminative conditioning of prism adaptation. Perception \& Psychophysics, 1971, 10, 90-92.

WELCH, R. B., \& RHOADES, R. W. The manipulation of informational feedback and its effects upon prism adaptation. Canadian Journal of Psychology, 1969, $23,415-428$.

(Accepted for publication May 17, 1972.) 